\title{
Development of Health mix from Lizard Fish and its Nutritional Characteristics
}

\author{
K. Rathnakumar ${ }^{1^{*}}$ and N. Pancharaja ${ }^{2}$ \\ ${ }^{1}$ Department of Fish Process Engineering, College of Fisheries Engineering, Tamil Nadu \\ Fisheries University, Nagapattinam-611002, Tamil Nadu, India \\ ${ }^{2}$ Department of Fisheries, Tenampet, DMS complex, Chennai, Tamil Nadu, India \\ *Corresponding author
}

\section{A B S T R A C T}

Keywords

Lizard fish, Acid hydrolysis, Health mix, Nutritional characteristics

Article Info

Accepted:

26 February 2018

Available Online:

10 March 2018
Minced meat fish Lizard fish (Saurida tumbil) was subjected to steam cooking and acid hydrolysis process and dried up to moisture content of 6$7 \%$. Fish powder obtained from both methods were incorporated in to a cereal mix containing malted and roasted wheat and ragi powder at different percentage $(10 \%, 15 \%, 20 \%$ and $25 \%)$ separately. Prepared health mix were stored at ambient temperature and its storage characteristics such as biochemical composition, nutritional, functional, microbiological parameters and organoleptical attributes were analyzed up to 90 days. Health drinks prepared by using Lizard fish powder by acid hydrolysis is more acceptable during the storage period.

\section{Introduction}

Protein deficiency, particularly animal protein deficiency is most common nutritional deficiency in India. Per capita protein availability in India is $58 \mathrm{~g} /$ day of which animal protein is only $9.5 \mathrm{~g}$ against the world average of $25 \mathrm{~g}$ (Thomas et al., 2006). Today, there is an ever increasing awareness about health foods and fish is finding acceptance for its special nutritional qualities. Rising wealth and a new focus on health foods are generating a firmer wave of demand for fish. About one billion people mostly in developing countries rely on fish as their primary animal protein source (Ferozkhan et al., 2006). To meet the increasing demand for fish, the under-utilized fish species are to be presented to the consumer as palatable attractive products.

In India, large quantities of low value fishes amounting to about $30 \%$ of the total marine catch are not properly utilized (Anon, 2001). Lizard fishes contributed about $5 \%$ of total demersal finfish landings in 2005 and its total landings in 30552 tonnes (CMFRI, 2006). Utilization of low value by catch for human consumption is mainly done in the form of mince based products. 
Fish can be used as a fortifying agent to improve the food value and taste of different food items. Malting of cereals gives number of advantages like increased phosphorus availability, vitamin-C and lysine content (Dulby and Tsai, 1976).The anti-nutritional factors such as phytase, trypsin inhibitor and haemagglutinins were broken down on germination (Reddy, et al., 1978). The present work was taken up to develop fish based health mix to convert locally abundant low value fishes to nutritionally rich edible product.

\section{Materials and Methods}

Lizard fish (Saurida tumbil) caught from Thoothukudi fishing harbour was used for present study. Minced meat were obtained using deboner (M/s. Baadar 601), washed and divided into two parts. One part is steam cooked for 10 minutes and another part is hydrolysed by using $1 \mathrm{~N} \mathrm{HCl} \mathrm{(Setty} \mathrm{et} \mathrm{al.,}$ 1977) and dried at $60^{\circ} \mathrm{C}$ until moisture content reaches 6-7\%. Both cooked and hydrolysed fish powder was mixed with cereal mix containing malted and roasted wheat and ragi powder (equal volume) at different percentage $(10 \%, 15 \%, 20 \%$ and $25 \%)$. Storage behaviors were studied up to 90 days.

Moisture, protein, fat, ash, peroxide value, (PV), calcium and pepsin digestibility were determined as per standard methods of AOAC (1995). Phosphorus was estimated by the method of Fiske and subburow (1925). Carbohydrate and available lysine were determined by the method of Sadasivam and Manickam (1992). Free fatty acids (FFA) were estimated by the method of Olley and Lovern (1960). TVB-N was estimated by the procedure of Beatty and Gibbons (1937) using Conway's micro diffusion technique. Fat absorption capacity (FAC) was estimated by the method of Lin et al., (1974). Water absorption capacity (WAC) was determined by the method of Solsulski (1962) and microbiological parameters such as TPC, $E$. coli, Staphylococci, Salmonella and Vibrio cholerae were determined by the method of APHA (1976). Organoleptical quality of health mix value evaluated by preparing a drink in boiling water with required amount of sugar and milk powder. Sensory quality such as appearance, colour, odour and overall acceptability were tested by trained panelist.

\section{Results and Discussion}

In the present study, the average length and weight of Lizard fish used was $22.6 \mathrm{~cm}$ and $93.6 \mathrm{~g}$ respectively. The yield of minced meat from whole fish was $50.0 \%$, Dora (1992) has reported that the dressing yield of $60 \%$ and $66 \%$ from croaker and pink perch respectively. Yield of fish meat powder is about $15.6 \%$ by cooking process and $15.0 \%$ by hydrolyzing process. The physico-chemical characteristics of fish vary with general factors such as season, size, maturity and climate. Physico-chemical, functional and microbiological characteristics of Lizard fish mince are presented in Table 1. TMA-N and PV was absent it is mainly due to repeated washing of meat at $4^{\circ} \mathrm{C}$. Higher solubility $(89.5 \%)$, viscosity $(12.85 \mathrm{cp})$ and $\mathrm{a} \mathrm{Ca}^{++}$ ATPase activity was $0.75 \mu \mathrm{g} \quad \mathrm{pi} / \mathrm{mg}$ protein/min indicated the freshness and conformational status of myofibrillar proteins.

Similar results reported by many workers (Numakura et al., 1989; Chan et al., 1995; Rathnakumar and Shanmasundar, 1998). Several authors have reported on the proximate composition and its nutritive value of Lizard fish (Srinivasan, 1966, Ismail et al., 1968 and Chattopadhyay et al., 2004). Fish meat had total plate count of $2.2 \times 10^{4} \mathrm{cfu} / \mathrm{g}$, Staphylococci and E. coli were found to be 8.0 x $10^{2} \mathrm{cfu} / \mathrm{g}$ and $3.0 \times 10^{2} \mathrm{cfu} / \mathrm{g}$ respectively. Salmonella and Vibrio were absent. 
Table.1 Physico-chemical functional \&microbiological characteristics of Lizard fish mince

\begin{tabular}{|c|c|c|}
\hline S. No & Parameters & $\begin{array}{l}\text { Saurida } \\
\text { tumbil }\end{array}$ \\
\hline 1. & Moisture (\%) & 76.99 \\
\hline 2. & Protein $(\%)$ & 19.01 \\
\hline 3. & Fat $(\%)$ & 1.13 \\
\hline 4. & Ash $(\%)$ & 1.31 \\
\hline 5. & TMA-N (mg \%) & Absent \\
\hline 6. & TVB-N (mg \%) & 1.4 \\
\hline 7. & $\operatorname{FFA}(\%$ of oleic acid $)$ & 0.0022 \\
\hline 8. & $\mathrm{PV}$ (milli equivalent $\mathrm{O}_{2} / \mathrm{kg}$ fat) & Absent \\
\hline 9. & NPN (mg/100g meat) & 194.52 \\
\hline 10. & $\mathrm{pH}$ & 7.01 \\
\hline 11. & Viscosity (Cp) & 12.85 \\
\hline 12. & Solubility (\%) & 89.51 \\
\hline 13. & $\begin{array}{l}\mathrm{Ca}^{2+} \mathrm{ATPase} \text { activity }((\mu \mathrm{g} \text { pi/mg } \\
\text { protein/min })\end{array}$ & 0.75 \\
\hline 14. & Total plate count (TPC) (cfu/g) & $2.2 \times 10^{4}$ \\
\hline 15. & Staphylococcus aureus (cfu/g) & $8.0 \times 10^{2}$ \\
\hline 16. & E.coli $(\mathrm{cfu} / \mathrm{g})$ & $3.0 \times 10^{2}$ \\
\hline 17. & Salmonella & Nil \\
\hline 18. & Vibrio cholerae & Nil \\
\hline
\end{tabular}

Table.2 Physico- chemical and functional characteristics of fish meat powder from lizard fish (Saurida tumbil)

\begin{tabular}{|c|c|c|c|}
\hline \multirow[t]{2}{*}{ Sl. No. } & \multirow[t]{2}{*}{ Parameters } & \multicolumn{2}{|c|}{ Lizard fish } \\
\hline & & $\begin{array}{c}\text { Cooked meat } \\
\text { powder }\end{array}$ & $\begin{array}{c}\text { Hydrolyzed meat } \\
\text { powder }\end{array}$ \\
\hline 1. & Moisture (\%) & 6.02 & 7.01 \\
\hline 2. & Protein $(\%)$ & 89.63 & 88.61 \\
\hline 3. & Fat $(\%)$ & 1.05 & 0.78 \\
\hline 4. & Ash (\%) & 2.06 & 3.06 \\
\hline 5. & Calcium (mg/100g) & 205.58 & 250.06 \\
\hline 6. & Phosphorus(mg/100g) & 287.54 & 296.37 \\
\hline 7. & Pepsin digestibility (\%) & 98.01 & 97.06 \\
\hline 8. & Available lysine(g/16g nitrogen) & 10.10 & 9.79 \\
\hline 9. & WAC (g water/g dried material) & 3.20 & 3.41 \\
\hline 10. & FAC(g oil/g dried material) & 1.00 & 1.20 \\
\hline 11. & TVB-N (mg \%) & 1.23 & 1.19 \\
\hline 12. & FFA (\% of oleic acid) & 0.009 & 0.007 \\
\hline 13. & PV (milli equivalent $\mathbf{O}_{2} / \mathrm{kg}$ fat) & 0.08 & 0.07 \\
\hline
\end{tabular}


Table.3 Changes in nutritional characteristics of cooked meat (Lizard fish) powder incorporated cereal mix

\begin{tabular}{|c|c|c|c|c|c|c|c|c|}
\hline Fish & \multicolumn{4}{|c|}{$10 \%$} & \multicolumn{4}{|c|}{$15 \%$} \\
\hline $\begin{array}{c}\text { Parameters } \\
\text { Storage } \\
\text { period (days) }\end{array}$ & $\begin{array}{l}\text { Calcium } \\
\text { (mg } \\
\text { /100g) }\end{array}$ & $\begin{array}{c}\text { Phosphorus } \\
\text { (mg/100g) }\end{array}$ & $\begin{array}{c}\text { Available } \\
\text { lysine (g/16 g } \\
\text { nitrogen) }\end{array}$ & $\begin{array}{c}\text { Pepsin } \\
\text { digestibility } \\
(\%)\end{array}$ & $\begin{array}{l}\text { Calcium } \\
\text { (mg } \\
\text { /100g) }\end{array}$ & $\begin{array}{c}\text { Phosphorus } \\
\text { (mg/100g) }\end{array}$ & $\begin{array}{c}\text { Available } \\
\text { lysine (g/16 } \\
\text { g nitrogen) }\end{array}$ & $\begin{array}{c}\text { Pepsin } \\
\text { digestibility } \\
(\%)\end{array}$ \\
\hline 0 & 190.63 & 291.86 & 6.59 & 88.95 & 191.73 & 292.94 & 6.80 & 90.01 \\
\hline 30 & 189.722 & 290.48 & 5.83 & 88.45 & 190.94 & 291.36 & 6.29 & 89.13 \\
\hline 60 & 188.80 & 289.10 & 5.06 & 87.96 & 190.15 & 289.79 & 5.79 & 88.26 \\
\hline 90 & 185.50 & 282.47 & 3.86 & 86.59 & 186.84 & 284.69 & 4.37 & $\mathbf{8 7 . 3 0}$ \\
\hline
\end{tabular}

\begin{tabular}{|c|c|c|c|c|c|c|c|c|}
\hline Fish & \multicolumn{4}{|c|}{$20 \%$} & \multicolumn{4}{|c|}{$25 \%$} \\
\hline $\begin{array}{c}\text { Parameters } \\
\begin{array}{c}\text { Storage } \\
\text { period } \\
\text { (days) }\end{array}\end{array}$ & $\begin{array}{c}\text { Calcium } \\
(\mathrm{mg} / 100 \mathrm{~g})\end{array}$ & $\begin{array}{c}\text { Phosphorus } \\
\text { (mg/100g) }\end{array}$ & $\begin{array}{c}\text { Available } \\
\text { lysine (g/16 } \\
\text { g nitrogen) }\end{array}$ & $\begin{array}{c}\text { Pepsin } \\
\text { digestibility } \\
(\%)\end{array}$ & $\begin{array}{c}\text { Calcium } \\
(\mathrm{mg} / 100 \mathrm{~g})\end{array}$ & $\begin{array}{c}\text { Phosphorus } \\
\text { (mg/100g) }\end{array}$ & $\begin{array}{c}\text { Available } \\
\text { lysine (g/16 } \\
\text { g nitrogen) }\end{array}$ & $\begin{array}{c}\text { Pepsin } \\
\text { digestibility } \\
(\%)\end{array}$ \\
\hline 0 & 192.83 & 293.41 & 7.14 & 91.11 & 193.58 & 294.37 & 7.50 & 92.10 \\
\hline 30 & 191.87 & 291.95 & 6.97 & 90.29 & 192.56 & 292.70 & 7.18 & 91.45 \\
\hline 60 & 190.92 & 290.50 & 6.81 & 89.48 & 191.54 & 291.03 & 6.86 & 90.80 \\
\hline 90 & 187.93 & 286.31 & 4.97 & 88.60 & 188.01 & 288.15 & 5.68 & 89.51 \\
\hline
\end{tabular}


Table.4 Changes in nutritional characteristics of hydrolysed meat (Lizard fish) powder incorporated cereal mix

\begin{tabular}{|c|c|c|c|c|c|c|c|c|}
\hline Fish meat & \multicolumn{4}{|c|}{$10 \%$} & \multicolumn{4}{|c|}{$15 \%$} \\
\hline $\begin{array}{c}\text { Parameters } \\
\text { Storage } \\
\text { period } \\
\text { (days) } \\
\end{array}$ & $\begin{array}{l}\text { Calcium } \\
(\mathrm{mg} / 100 \mathrm{~g})\end{array}$ & $\begin{array}{c}\text { Phosphorus } \\
\text { (mg/100g) }\end{array}$ & $\begin{array}{c}\text { Available } \\
\text { lysine (g/16 } \\
\text { g nitrogen) }\end{array}$ & $\begin{array}{c}\text { Pepsin } \\
\text { digestibility } \\
(\%)\end{array}$ & $\begin{array}{c}\text { Calcium } \\
(\mathrm{mg} / 100 \mathrm{~g})\end{array}$ & $\begin{array}{l}\text { Phosphorus } \\
(\mathrm{mg} / 100 \mathrm{~g})\end{array}$ & $\begin{array}{c}\text { Available } \\
\text { lysine (g/16 } \\
\text { g nitrogen) }\end{array}$ & $\begin{array}{c}\text { Pepsin } \\
\text { digestibility } \\
(\%)\end{array}$ \\
\hline 0 & 194.03 & 293.49 & 6.40 & 88.57 & 197.85 & 294.55 & 6.68 & 89.64 \\
\hline 30 & 193.38 & 291.25 & 6.29 & 88.46 & 197.21 & 292.51 & 6.44 & 89.26 \\
\hline 60 & 192.75 & 289.01 & 6.18 & 88.36 & 196.58 & 290.47 & 6.20 & 88.89 \\
\hline 90 & 190.10 & 287.80 & 4.27 & 87.50 & 192.81 & 289.20 & 5.11 & 87.69 \\
\hline
\end{tabular}

\begin{tabular}{|c|c|c|c|c|c|c|c|c|}
\hline Fish meat & \multicolumn{4}{|c|}{$20 \%$} & \multicolumn{4}{|c|}{$25 \%$} \\
\hline $\begin{array}{c}\text { Parameters } \\
\text { Storage } \\
\text { period } \\
\text { (days) }\end{array}$ & $\begin{array}{c}\text { Calcium } \\
(\mathrm{mg} / 100 \mathrm{~g})\end{array}$ & $\begin{array}{c}\text { Phosphorus } \\
\text { (mg/100g) }\end{array}$ & $\begin{array}{c}\text { Available } \\
\text { lysine (g/16 } \\
\text { g nitrogen) }\end{array}$ & $\begin{array}{c}\text { Pepsin } \\
\text { digestibility } \\
(\%)\end{array}$ & $\begin{array}{l}\text { Calcium } \\
(\mathrm{mg} / 100 \mathrm{~g})\end{array}$ & $\begin{array}{c}\text { Phosphorus } \\
\text { (mg/100g) }\end{array}$ & $\begin{array}{c}\text { Available } \\
\text { lysine (g/16 } \\
\text { g nitrogen) }\end{array}$ & $\begin{array}{c}\text { Pepsin } \\
\text { digestibility } \\
(\%)\end{array}$ \\
\hline 0 & 201.79 & 295.71 & 6.71 & 90.75 & 204.09 & 295.21 & 6.77 & 91.70 \\
\hline 30 & 200.50 & 294.03 & 6.56 & 90.16 & 203.59 & 294.35 & 6.62 & 91.15 \\
\hline 60 & 199.20 & 292.36 & 6.41 & 89.57 & 203.10 & 293.50 & 6.47 & 90.60 \\
\hline 90 & 197.26 & 290.48 & 5.21 & 88.80 & 201.15 & 291.10 & 5.46 & 89.48 \\
\hline
\end{tabular}


Table.5 Changes in the functional characteristics of cooked meat (lizard fish) powder incorporated cereal mix

\begin{tabular}{|c|c|c|c|c|c|c|c|c|}
\hline $\begin{array}{c}\text { Fish meat } \\
\text { powder }\end{array}$ & \multicolumn{2}{|c|}{$10 \%$} & \multicolumn{3}{c|}{$15 \%$} & \multicolumn{2}{c|}{$20 \%$} & \\
\hline \begin{tabular}{c} 
Parameters \\
\hline $\begin{array}{c}\text { Storage } \\
\text { period } \\
\text { (days) }\end{array}$
\end{tabular} & WAC & FAC & WAC & FAC & WAC & FAC & WAC & FAC \\
\hline 0 & 2.50 & 0.67 & 2.58 & 0.70 & 2.65 & 0.71 & 2.72 & 0.73 \\
\hline \hline 30 & 2.58 & 0.62 & 2.65 & 0.65 & 2.72 & 0.68 & 2.79 & 0.70 \\
\hline 60 & 2.66 & 0.56 & 2.73 & 0.61 & 2.79 & 0.65 & 2.86 & 0.68 \\
\hline 90 & 2.54 & 0.50 & 2.64 & 0.57 & 2.71 & 0.62 & 2.78 & 0.65 \\
\hline
\end{tabular}

WAC - Water absorption capacity (g water/g dried material)

$\mathrm{FAC}-\mathrm{F}$ at absorption capacity (g oil / g dried material)

Table.6 Changes in the functional characteristics of hydrolysed meat (Lizard fish) powder incorporated cereal mix

\begin{tabular}{|c|c|c|c|c|c|c|c|c|}
\hline $\begin{array}{c}\text { Fish meat } \\
\text { powder }\end{array}$ & \multicolumn{2}{|c|}{$10 \%$} & \multicolumn{3}{c|}{$15 \%$} & \multicolumn{3}{c|}{$20 \%$} \\
\hline $\begin{array}{c}\text { Parameters } \\
\begin{array}{c}\text { Storage } \\
\text { period } \\
\text { days) }\end{array}\end{array}$ & WAC & FAC & WAC & FAC & WAC & FAC & WAC & FAC \\
\hline 0 & 2.62 & 0.69 & 2.69 & 0.72 & 2.75 & 0.75 & 2.84 & 0.78 \\
\hline 30 & 2.70 & 0.66 & 2.77 & 0.69 & 2.83 & 0.73 & 2.95 & 0.76 \\
\hline 60 & 2.78 & 0.63 & 2.85 & 0.67 & 2.92 & 0.71 & 3.00 & 0.73 \\
\hline 90 & 2.70 & 0.60 & 2.74 & 0.64 & 2.80 & 0.68 & 2.87 & 0.71 \\
\hline
\end{tabular}

WAC - Water absorption capacity (g water/g dried material)

$\mathrm{FAC}-\mathrm{F}$ at absorption capacity ( $\mathrm{g}$ oil / $\mathrm{g}$ dried material)

Table.7 Changes in biochemical of cooked meat (Lizard Fish) incorporated powder cereal mix

\begin{tabular}{|c|c|c|c|c|c|c|c|c|c|c|c|c|}
\hline $\begin{array}{l}\text { Fish meat } \\
\text { powder }\end{array}$ & \multicolumn{3}{|c|}{$10 \%$} & \multicolumn{3}{|c|}{$15 \%$} & \multicolumn{3}{|c|}{$20 \%$} & \multicolumn{3}{|c|}{$25 \%$} \\
\hline Parameters & & PV & TVB-N & FFA & PV & TVB- & FFA & PV & TVB-N & & PV & TVB- \\
\hline $\begin{array}{l}\text { Storage } \\
\text { period } \\
\text { (days) }\end{array}$ & FFA & & & & & $\mathrm{N}$ & & & & FFA & & \\
\hline 0 & 0.04 & 0.37 & 1.55 & 0.04 & 0.35 & 3.18 & 0.04 & 0.32 & 4.81 & 0.03 & 0.29 & 6.45 \\
\hline 30 & 0.05 & 0.51 & 3.79 & 0.05 & 0.49 & 5.35 & 0.05 & 0.46 & 6.96 & 0.04 & 0.41 & 8.10 \\
\hline 60 & 0.06 & 0.65 & 6.04 & 0.06 & 0.63 & 7.51 & 0.06 & 0.59 & 9.12 & 0.05 & 0.52 & 9.75 \\
\hline 90 & 0.08 & 0.82 & 10.36 & 0.07 & 0.78 & 13.01 & 0.07 & 0.77 & 14.97 & 0.07 & 0.70 & 16.58 \\
\hline
\end{tabular}

FFA-Free Fatty Acid (\%Oleic acid), PV-Peroxide Value (milli equivalent of $\mathrm{O}_{2} / \mathrm{kg}$ of fat),

TVB-N - Total Volatile Base -Nitrogen (mg \%). 
Table.8 Changes in biochemical characteristics in hydrolysed meat (Lizard Fish) powder incorporated cereal mix

\begin{tabular}{|c|l|l|l|l|l|l|l|l|l|l|l|l|}
\hline $\begin{array}{c}\text { Fish } \\
\text { powder }\end{array}$ & \multicolumn{3}{|c|}{$10 \%$} & \multicolumn{3}{c|}{$15 \%$} & \multicolumn{3}{c|}{$20 \%$} & & $25 \%$ \\
\hline $\begin{array}{c}\text { Parameters } \\
\begin{array}{c}\text { Storage } \\
\text { period } \\
\text { (days) }\end{array}\end{array}$ & FFA & PV & TVB-N & FFA & PV & $\begin{array}{c}\text { TVB- } \\
\text { N }\end{array}$ & FFA & PV & TVB-N & FFA & PV & TVB- \\
\hline 0 & 0.04 & 0.16 & 1.06 & 0.04 & 0.14 & 2.30 & 0.04 & 0.13 & 3.55 & 0.03 & 0.11 & 5.16 \\
\hline $\mathbf{3 0}$ & 0.05 & 0.32 & 2.61 & 0.05 & 0.30 & 4.41 & 0.04 & 0.26 & 6.10 & 0.04 & 0.24 & $\mathbf{8 . 0 6}$ \\
\hline $\mathbf{6 0}$ & 0.06 & 0.47 & 4.16 & 0.06 & 0.45 & 6.53 & 0.05 & 0.40 & 8.67 & 0.05 & 0.37 & $\mathbf{1 0 . 9 7}$ \\
\hline 90 & $\mathbf{0 . 0 7}$ & $\mathbf{0 . 6 6}$ & $\mathbf{8 . 3 7}$ & $\mathbf{0 . 0 7}$ & $\mathbf{0 . 6 2}$ & $\mathbf{1 1 . 2 7}$ & $\mathbf{0 . 0 6}$ & $\mathbf{0 . 5 9}$ & $\mathbf{1 4 . 7 5}$ & $\mathbf{0 . 0 6}$ & $\mathbf{0 . 5 4}$ & $\mathbf{1 7 . 7 9}$ \\
\hline
\end{tabular}

FFA-Free Fatty Acid (\%Oleic acid), PV-Peroxide Value (milli equivalent of $\mathrm{O}_{2} / \mathrm{kg}$ of fat),

TVB-N - Total Volatile Base -Nitrogen (mg \%).

The physico-chemical and functional properties of fish meat powder from Lizard fish meat prepared by both steam cooking and hydrolyzing process is given in Table 2 . The protein content of fish powder from cooked and hydrolyzed meat powder was $89.63 \%$ and $88.61 \%$ respectively. Available lysine and pepsin digestibility of above powders were $9.94 \%$ and $9.62 \%$ respectively. The results of the present study are in agreement with previous workers (Setty et al., 1977). Calcium and phosphorus content of cooked meat powder were found to be $205.58(\mathrm{mg} / 100 \mathrm{~g})$ and $287.54(\mathrm{mg} / 100 \mathrm{~g})$ respectively, whereas in hydrolysed meat powder it was 250.06 and $296.37(\mathrm{mg} / 100 \mathrm{~g})$ respectively. The values of TVB-N, FFA and PV were found to be 1.23 (mg \%), $0.009 \%$ of oleic acid and 0.08 milli equivalent $\mathrm{O}_{2} / \mathrm{kg}$ of fat respectively in cooked meat powder, also as in hydrolysed meat powder it was found to be $1.19 \mathrm{mg} \%$, $0.007 \%$ of oleic acid and 0.07 milli equivalent $\mathrm{O}_{2} / \mathrm{kg}$ of fat respectively. TVB-N, FFA and PV values of hydrolysed meat powder were less than cooked meat powder, it may be due to hydrolysis process. The values of TVB-N, FFA and PV in the present study were less than the result reported by chattopadhyay et al., (2004) in edible meat from Silverbellies. During storage period proximate composition did not vary significantly in both cooked as well as hydrolysed meat powder incorporated with cereals at different percentage. However protein content increased with increase in amount of fish powder similarly carbohydrate value was decreased. The highest protein content $(34.2 \%)$ was found in $25 \%$ cooked fish powder incorporated mix, where as it was $29.60 \%$ in hydrolysed meat powder incorporated mix. The results for changes in nutritional characteristics of both health mix are presented Table 3 and 4 . The values of available lysine and pepsin digestibility were found to increase with increasing amount of fish powder incorporation. The highest value of available lysine $\left(7.50 \mathrm{~g} / 16 \mathrm{gN}_{2}\right)$ and pepsin digestibility $(92.1 \%)$ were found in $25 \%$ cooked meat powder incorporated mix, whereas in hydrolysed meat powder incorporated mix it was 6.77 (g/10g nitrogen) and $91.70 \%$ respectively. There is significant difference $(\mathrm{P}<0.05)$ found in the nutritional characteristics of health mix. The value of calcium and phosphorus are also increases with increasing amount of fish powder incorporation. During storage slight reduction in the value of available lysine and pepsin digestibility were observed. Similar trend were observed by Rehman (2006) in cereals. The results for changes in functional properties of health mix are presented in Table 5 and 6 . The functional properties such as WAC and FAC were found to be 
increasing trend with increasing amount of fish powder added. During storage as slight increase in WAC were found. This is may be due to fact that denatured proteins bind more water through exposure of the interior hydrophilic groups (Kinsella, 1982). The results for changes in biochemical characteristics are presented in Table 7 and 8. The value of TVB-N, PV, and FFA were increased during storage period. FFA and TVB-N and PV content vary significantly $(\mathrm{P}<0.05)$ during storage period in cooked meat powder incorporated mix. FFA content did not vary significantly during storage period in hydrolysed meat powder incorporated mix whereas PV and TVB-N did vary at $1 \%$ and $5 \%$ respectively.

Initial TPC value was $3.8 \times 10^{3} \mathrm{cfu} / \mathrm{g}$ and after 90 days it was $1.9 \times 10^{3} \mathrm{cfu} / \mathrm{g}$ in cooked meat powder incorporated mix, and it was $4.0 \times 10^{3}$ $\mathrm{cfu} / \mathrm{g}$ and after 90 days $2.0 \times 10^{3} \mathrm{cfu} / \mathrm{g}$ in hydrolysed meat powder incorporated mix respectively. Reduction in the TPC is mainly due to low water activity. Salmonella, $V$. cholerae and $E$. coli were absent throughout the storage period. Health drink was prepared by mixing $10-15 \mathrm{~g}$ of mix in required amount of hot water with sugar and milk powder. Organoleptic quality revealed that the tastes of the liquid drinks prepared were sweet in taste and no fishy odour immediately after its preparation for different study period. However there is significant difference $(p<0.01)$ observed in their overall acceptability.

The drink had no fishy odour and sweet in taste. Drink prepared from hydrolyzed meat powder - cereal mixture was more acceptable than it is prepared using cooked fish meat powder - cereal mixture. Compare to four different ratios tried $10 \%$ hydrolyzed meat powder - cereal mixture shows better organoleptic acceptability throughout the study.

\section{References}

Anon., 2001. Indian marine product rises again. Seafood export Journal., 32(6): 7-12. CMFRI. 2006. Central Marine Fisheries Research Institute, Annual report 2005-06, ICAR, India.

AOAC, 1995. Official method of Analysis, $16^{\text {th }}$ edn. Association of official analytical chemists, Washington, D.C.

APHA, 1976. Compendium of methods for the microbiological examination of foods, Speck M.L., (ed), APHA, New York.

Beatty*, S.A., and Gibbons., 1937. The measurement of spoilage in fish. J. Biol Board.Can., 3(1): 77-91.

Chan, J.K., and Gill, T.A., Thompson, J.N. and Singer., 1995. Herring surimi during low temperature setting, physico-chemical and texture properties. J.Food Sci., 66:1248-1253

Chattopadhyay, A.K., Madhusudana Roa, B., and Gupta, S., 2004. A simple process for the utilization of small bony fish as edible fish powder. Fish Technol., 41(2): 117-120.

Dulby, A., and Tsai, C.Y., 1976. Lysine and tryptophan increases during germination of cereal grains. Cereal Chemistry. 53:222-224.

Ferozkhan, M., and Panikkar, P., 2006. Indian Fisheries scenario: A peep into Constraints / Needs for its future development. Fishing chimes., 26(9):13-20.

Fiske, C.H., and Subbarow, Y., 1925. The colorimetric determination of phosphorus. J. Biol. Chem, 66: 375-380.

Kinsella, J.E., 1982. Relation between structural and functional properties of food protein. In: Food Proteins, Fox, R.F. and Condon, J.J., (eds). Applied science publishers, Newyork. pp. 51103. 
Lin, M.Y.J., Humert, E.S. and Sosulski, F.W., 1974. Certain functional properties of Sunflower meal product. J. Food Sci., 39: 368-370.

Numakura, T., Mizoguchi, R., Kimura, I., Toyoda, K., Fujita, T., Seki, N., Arai,K.,1989. Changes in gel forming ability and cross-linking ability of myosin chain of Alaska bollack surimi denatured by heat treatment. Nippon Suisan Gakkaishi., 55:1083-1090.

Olley, T., and Lovern, J.A., 1960. Phospholipid hydrolysis of cod flesh stored at various temperature, J. Sci. Food Agric., 11(11): 644-652.

Rathnakumar, K.and Shamasundar, B.A., 1998. Visco - elastic properties of pinkperch (Nemipterus japonicus) during setting and gelation. In:Advances and Priorities Fisheries Technology. Balachandran, K.K., Iyer, T.S.G., Madhavan, P., Joseph, J., Perigreen, P.A., Raghunath, M.R. and Varghese, M.D. (eds), Society of Fisheries Technologists (India), Cochin. pp. 250-256.

Reddy, N.R., Balakrishnan, and Salunkhe, D.K., 1978. Phytate, phosphorus and mineral changes during germination. $J$. Food Science., 43: 540-543.

Sadasivam, S., and Manickam, A., 1992 (b). Esstimatiion of Lysine. In: Biochemical methods for Agricultural Sciences. Wiley Eastern Limited, New Delhi: pp. 45-46.

Setty, R.T.M., Sudhakara, N.S., Nagaraj, A.S., and Shetty, H.P.C., 1977. Development of partially hydrolysed and deodourised fish powder. Fish Technol., 14(2): 103-108.

Setty, R.T.M., Sudhakara, N.S., Nagaraj, A.S., and Shetty, H.P.C., 1977. Development of partially hydrolysed and deodourised fish powder. Fish Technol., 14(2): 103-108.

Solsulski, F.W., 1962. The centrifuge method for determining water absorption in hard red spring wheats. Cereal Chem., 39: 344-350.

Thomas, R., Anjaneyulu, A.S.R., and Kondiah, N., 2004. Soy proteins: Use in meat products and their impact on human health. Indian Food Industry., 23(1):42-44.

\section{How to cite this article:}

Rathnakumar, K. and Pancharaja, N. 2018. Development of Health mix from Lizard Fish and its Nutritional Characteristics. Int.J.Curr.Microbiol.App.Sci. 7(03): 3136-3144. doi: https://doi.org/10.20546/ijcmas.2018.703.362 